\title{
A function-based screen for seeking RubisCO active clones from metagenomes: novel enzymes influencing RubisCO activity
}

\author{
Stefanie Böhnke and Mirjam Perner \\ Molecular Biology of Microbial Consortia, Biocenter Klein Flottbek, University of Hamburg, Hamburg, \\ Germany
}

\begin{abstract}
Ribulose-1,5-bisphosphate carboxylase/oxygenase (RubisCO) is a key enzyme of the Calvin cycle, which is responsible for most of Earth's primary production. Although research on RubisCo genes and enzymes in plants, cyanobacteria and bacteria has been ongoing for years, still little is understood about its regulation and activation in bacteria. Even more so, hardly any information exists about the function of metagenomic RubisCOs and the role of the enzymes encoded on the flanking DNA owing to the lack of available function-based screens for seeking active RubisCOs from the environment. Here we present the first solely activity-based approach for identifying RubisCO active fosmid clones from a metagenomic library. We constructed a metagenomic library from hydrothermal vent fluids and screened 1056 fosmid clones. Twelve clones exhibited RubisCO activity and the metagenomic fragments resembled genes from Thiomicrospira crunogena. One of these clones was further analyzed. It contained a $35.2 \mathrm{~kb}$ metagenomic insert carrying the RubisCO gene cluster and flanking DNA regions. Knockouts of twelve genes and two intergenic regions on this metagenomic fragment demonstrated that the RubisCO activity was significantly impaired and was attributed to deletions in genes encoding putative transcriptional regulators and those believed to be vital for RubisCO activation. Our new technique revealed a novel link between a poorly characterized gene and RubisCO activity. This screen opens the door to directly investigating RubisCO genes and respective enzymes from environmental samples.
\end{abstract}

The ISME Journal (2015) 9, 735-745; doi:10.1038/ismej.2014.163; published online 9 September 2014

\section{Introduction}

Among the autotrophic $\mathrm{CO}_{2}$ fixation pathways, the Calvin Benson (CB) cycle accounts for most of Earth's net primary production ( $>99.5 \%$ of $105 \times 10^{9}$ tons per year) (Field et al., 1998; Raven, 2013). One of its key enzymes is the ribulose-1,5-bisphosphate carboxylase/ oxygenase (RubisCO, EC 4.1.1.39), which can catalyze the carboxylation of ribulose-1,5-bisphosphate to 3 phosphoglycerate. Four types of structural RubisCOs are currently distinguished, of which two types, form I (CbbLS) and form II (CbbM), are known to operate in the classical autotrophic CB cycle (Tabita et al., 2007). RubisCO is present in all plants, cyanobacteria and many autotrophic bacteria (Berg, 2011) and is believed to be the most abundant protein on Earth (Ellis, 1979; Raven, 2009). Hence, the CB cycle and the RubisCO enzymes are of fundamental importance for global primary production and it is vital to better understand RubisCO evolution and its diversity.

Correspondence: M Perner, Molecular Biology of Microbial Consortia, Biocenter Klein Flottbek, University of Hamburg, Ohnhorststr. 18, 22609 Hamburg, Germany.

E-mail: mirjam.perner@uni-hamburg.de

Received 23 April 2014; revised 15 July 2014; accepted 11 August 2014; published online 9 September 2014
Despite multiple years of research on RubisCO genes and enzymes (Li et al., 1993; Portis, 2003; Mueller-Cajar et al., 2011), still comparatively little knowledge exists about their regulatory mechanisms, assembly or activation. The role that CbbQ (AAA + ATPase domain) and CbbO (von Willebrand factor, type A) play in prokaryotic RubisCO activation is still enigmatic. In some cases, they are likely involved in post-translational RubisCO activation (Hayashi et al., 1997, 1999), whereas in other cases they do not appear to influence RubisCO activity at all (Schwedock et al., 2004). Also, the information on the mechanisms that regulate RubisCO expression is scarce: transcriptional regulators of the LysR family have been found in many genomes adjacent to the structural RubisCO genes (Kusian and Bowien, 1997; Scott et al., 2006). In the Hydrogenovibrio marinus genome two of these regulatory proteins, namely CbbR1 and CbbRm, are encoded upstream of the RubisCO structural genes $c b b L S-1$ and $c b b M$, respectively, and have been demonstrated to regulate adjacent RubisCO gene expression (Toyoda et al., 2005).

Most of our understanding of RubisCO functioning is based on the studies conducted with cultured bacteria (Joshi et al., 2009 and references therein; 
Tabita et al., 2008 and references therein), but hardly any information exists about the function of environmental RubisCOs and the role of the enzymes encoded on the flanking DNA regions. Approaches that are currently used for gathering information on environmental RubisCO gene clusters rely on sequence searches (for example, Xie et al., 2011). In some cases this has been advanced to subsequently determine the recombinant RubisCO activity in a surrogate host after having identified the RubisCO gene through sequence similarity first (Witte et al., 2010). However, inherent limitations of sequence-based screening exist because only sequences with significant similarities to known genes can be detected. To date, there is no method available that allows seeking RubisCOs by a function-based approach alone on a large scale directly from environmental DNA (metagenomic libraries). Therefore, the large majority of RubisCO gene clusters from uncultured organisms (>99\%) remains inaccessible and the questions whether the environmental RubisCOs are functional and how they are regulated and activated remain largely unanswered. We here describe a novel functionbased approach whereby RubisCO active enzymes can be sought from the large majority of uncultured organisms inhabiting the environment. We constructed a metagenomic fosmid library from deepsea hydrothermal fluids and screened the clones for recombinant RubisCO activity. The metagenomic DNA of fosmid clones exhibiting RubisCO activity was then further analyzed to elucidate the role of flanking genes and resulting gene products fundamental for expressing a fully functional RubisCO enzyme.

\section{Materials and methods}

Growth of TH-55 and cloning of RubisCO genes Thiomicrospira crunogena TH-55 (DSMZ No. 12353) was obtained from the German Collection of Microorganisms and Cell Cultures (DSMZ, Braunschweig, Germany) and was cultivated as described (Jannasch et al., 1985; Dobrinski et al., 2005). Cells were harvested after $24 \mathrm{~h}$, DNA was extracted and RubisCO form I ( $c b b L S)$ and form II $(c b b M)$ encoding gene regions were amplified (incorporating at least $500 \mathrm{bp}$ upstream to include possible promoter regions) and cloned into the fosmid vector pCC1FOS according to standard procedures (see Supplementary Materials and Methods).

\section{Sampling of hydrothermal fluids}

The hydrothermal fluids were collected from the Nibelungen field at $8^{\circ} 18^{\prime} \mathrm{S}$ at a water depth of $2915 \mathrm{~m}$ along the Mid Atlantic Ridge (Melchert et al., 2008). The investigated low-temperature hydrothermal fluids originated from the interface between hot fluids emanating from a crater and the ambient seawater. During sampling the onlinemonitored fluid temperature was between 90 and $120^{\circ} \mathrm{C}$ (Perner et al., 2013). The fluids had a $\mathrm{pH}$ of 5.6, $22 \mu \mathrm{M}$ hydrogen, $8 \mu \mathrm{M}$ sulfide and $56 \mu \mathrm{M}$ oxygen (Perner et al., 2013). Sampling was done by a remote-operated vehicle (ROV 6000, GEOMAR, Kiel) during the MAR-SUED V cruise (March/April 2009) with the RV Meteor. Around $200 \mathrm{ml}$ of hydrothermal fluids were concentrated onboard on a $0.2 \mu \mathrm{m}$ polycarbonate filter and kept at $-20^{\circ} \mathrm{C}$. Detailed sampling procedures and information on the sampling site are described elsewhere (Perner et al., 2007, 2009, 2013).

\section{Construction of fosmid libraries}

Two fosmid libraries were constructed: (i) one genome library with DNA material from TH-55 and (ii) one metagenomic library with the hydrothermal fluids from Nibelungen. Treatment of TH-55 and environmental DNA prior to metagenomic fosmid library construction is reported in Supplementary Materials and Methods. The (meta)-genome libraries were constructed using the pCC1FOS fosmid vector of the CopyControl Fosmid Library Production Kit (Epicentre, Madison, WI, USA) according to the manufacturer's instructions. Fosmid clones were selected on Luria-Broth (LB) agar plates by blue-white screening and chloramphenicol $\left(12.5 \mu \mathrm{g} \mathrm{ml}^{-1}\right)$. The quality of fosmid inserts was randomly checked and DNA inserts were sequenced (Supplementary Materials and Methods).

\section{Transposon mutagenesis}

Two fosmid transposon insertion libraries were constructed with RubisCO active metagenomic fosmid clones using the EZ-Tn $5^{\mathrm{TM}}<\mathrm{KAN}-2>$ insertion kit (Epicentre): (i) one with the fosmid clone 71C2 $(35.2 \mathrm{~kb})$ carrying the RubisCO gene cluster (cbbQOM lysR2 lysR1 cbbLSQO) and flanking DNA regions and (ii) one with the fosmid subclone 71C2II, comprising only the RubisCO gene cluster (cbbQOM lysR2 lysR1 cbbLSQO) (13 kb). The 71C2 and 71C2II transposon libraries consisted of 360 and 24 clones, respectively, which were sequenced with the manufacturer's designed KAN-2 FP-1 forward primer targeting the kanamycin cassette (5'-ACCTA CAACAAAGCTCTCATCAACC-3 ${ }^{\prime}$ ) to identify the insertion position. Transposon clones were selected by chloramphenicol $\left(12.5 \mu \mathrm{g} \mathrm{ml}^{-1}\right)$ and kanamycin $\left(50 \mu \mathrm{g} \mathrm{ml}^{-1}\right)$. Selected clones were tested for RubisCO activity by following the high-pressure liquid chromatography (HPLC) based activity assay described below.

\section{Preparation of crude extracts}

Pure cultures of TH-55 were harvested at the end of the exponential growth phase by centrifugation 
(17 600 g, $20 \mathrm{~min}, 4^{\circ} \mathrm{C}$ ). Escherichia coli clones with pCC1FOS::cbbLS and pCC1FOS::cbbM as well as single metagenomic fosmid and transposon clones were grown at $28{ }^{\circ} \mathrm{C}$ on $200 \mathrm{ml}$ preheated LB medium with chloramphenicol $\left(12.5 \mu \mathrm{g} \mathrm{ml}^{-1}\right.$ ) (and for transposon clones additionally with kanamycin $\left.\left(50 \mu \mathrm{g} \mathrm{ml}^{-1}\right)\right)$ and autoinduction solution $(1 \times$ final concentration (Epicentre)) in $1 \mathrm{l}$ flasks at 130 r.p.m. All these E. coli cells were harvested after $18 \mathrm{~h}$ by centrifugation $\left(9800 \mathrm{~g}, 10 \mathrm{~min}, 4^{\circ} \mathrm{C}\right.$ ). After centrifugation, cell pellets were washed twice with buffer $\mathrm{A}$ (100 mm Tris-HCl (pH 7.8), $10 \mathrm{~mm} \mathrm{MgCl}_{2}, 1 \mathrm{~mm}$ EDTA, $25 \mathrm{~mm} \mathrm{NaHCO}_{3}$ and $1 \mathrm{~mm}$ DTT) and resuspended in $2 \mathrm{ml}$ of the same buffer. Cells were fractured by French press, followed by centrifugation (19580 g, $\left.20 \mathrm{~min}, 4^{\circ} \mathrm{C}\right)$. Supernatants were used for analyzing the specific activity of RubisCO. The concentration of total protein in the crude extracts was measured according to Bradford and Williams (1976).

To upscale the RubisCO activity screen we tested whether one RubisCO active fosmid with TH-55 genomic DNA (clone 6F3) could still be detected among 11, 23, 47 and 95 inactive clones. The RubisCO inactive fosmid clone was $6 \mathrm{~F} 8$ (also from the genomic TH-55 library). These RubisCO active and inactive clones were grown together on one LB agar plate $\left(12.5 \mu \mathrm{g} \mathrm{ml}^{-1}\right.$ chloramphenicol) overnight at $37^{\circ} \mathrm{C}$. The metagenomic fosmid clones were then swamped off with $10 \mathrm{ml}$ LB medium $\left(12.5 \mu \mathrm{g} \mathrm{ml}^{-1}\right.$ chloramphenicol), and $200 \mu \mathrm{l}$ of this cell suspension was used as inoculum for the working cultures, grown as described above. For identifying RubisCO active fosmid clones from the Nibelungen metagenomic library, pools of 24 metagenomic clones were screened and prepared as described for the other pooled samples. If RubisCO activity was confirmed in the pool of 24 fosmid clones, then two pools each consisting of 12 fosmid clones were prepared as described above and tested for RubisCO activity. The pool with activity was then further broken down into three pools each containing four fosmid clones and RubisCO activity was measured. The four remaining fosmid clones from the pool with the activity were then screened individually for activity.

\section{RubisCO activity assay}

The enzyme assay was performed at $25^{\circ} \mathrm{C}$ for individual cultures or at 25 and $55^{\circ} \mathrm{C}$ if testing pools for RubisCO activity. The assay mixture (150 $\mu \mathrm{l}$ final volume) contained buffer A, $0.2 \mathrm{mg}$ unpurified, total protein and $5 \mathrm{~mm}$ ribulose-1,5bisphosphate (Rubp) - Rubp addition is the initiation step. If RubisCO enzymes are in the sample Rubp is converted to 3-phosphoglycerate (3-PGA) over time. To monitor this conversion, subsamples $(50 \mu \mathrm{l})$ were taken at different time points $(0,30$ and $120 \mathrm{~min}$ for pools and 0,10 and $30 \mathrm{~min}$ for single active clones) from the assay mixture. The reactions were stopped at $95{ }^{\circ} \mathrm{C}$ for $3 \mathrm{~min}$ and denatured proteins removed by centrifugation (16100g, $20 \mathrm{~min}, 4^{\circ} \mathrm{C}$ ). The supernatant was used to quantify the concentration of Rubp and 3-PGA with HPLC (LaChrom Elite system, Hitachi, Tokyo, Japan) with a Lichrospher 100 RP-18e column (VWR International GmbH, Darmstadt, Germany). HPLC procedures were performed as described (Jakob and Saenger, 1985) with some modifications: detection was at $200 \mathrm{~nm}$, with acetonitrile as an eluent and a flow of $0.6 \mathrm{ml} \mathrm{min}^{-1}$.

Transcription experiments with TH-55 and transposon clones

Total RNA from (i) TH-55 was isolated with the Presto Mini RNA Bacteria Kit (Geneaid, New Taipei City, Taiwan) and (ii) from the metagenomic fosmid clones 71C2, the subcloned 71C2II and the transposon clones 96 $17 \mathrm{II} \Delta c b b O-\mathrm{m}$ using the UltraClean Microbial RNA Isolation Kit (MO BIO Laboratories, Inc., Carlsbad, CA, USA). Genomic DNA was removed (RTS DNase Kit, MO BIO Laboratories, Inc.) and total cDNA synthesized (Invitrogen's SuperScript VILO cDNA Synthesis Kit, Life Technologies, Darmstadt, Germany). cDNA served as a template for the amplification of (i) different gene-to-gene interspaces to elucidate which genes are co-transcribed in TH-55 and (ii) small fragments of $\sim 300 \mathrm{bp}$ within $c b b L$ and $c b b M$ to examine the relative variations of transcript amounts of RubisCO form I and II structural genes in the above-mentioned transposon clones and respective intact versions. Primers used for amplifications are listed in Supplementary Table S1. Controls of the template lacking reverse transcriptase were performed by PCR. For details on culturing and RT-PCR conditions see Supplementary Materials and Methods.

Complementation experiments

To test whether the activity of the transposon clones could be restored, transposon clones 96 $\Delta$ orf06, 149II $\Delta$ lysR2, 6II $\Delta l y s R 1$ and $17 \mathrm{II} \Delta c b b O-m$ were complemented with pUC19::orfo6, pUC19::lysR2, pUC19::lysR1 and pUC19::cbbO-m, respectively. For detailed procedures see Supplementary Materials and Methods.

Sequence access

The nucleotide sequence of the entire $35.2 \mathrm{~kb}$ metagenomic fragment has been submitted to the GenBank database under accession number KJ639815.

\section{Results and discussion}

Establishing a function-based screen for detecting RubisCO active fosmid clones

We used a HPLC-based approach to establish a new functional screen for seeking RubisCO active 
recombinant enzymes from metagenomic fosmid clones (for experimental setup see Figure 1). First, we tested our screen by measuring RubisCO activity from crude extracts of TH-55 and from recombinantly expressed RubisCOs of TH-55. Three types of cloned RubisCO genes and gene clusters from TH-55 were used for the initial tests: one consisted of a large genomic DNA fragment containing $38.1 \mathrm{~kb}$ and included the RubisCO gene cluster ( $c b b Q O M$ lysR2 lysR1 cbbLSQO) and flanking regions $(25.1 \mathrm{~kb})$ (pCC1FOS fosmid clone 6F3), the second encoded only the small and large subunit of the RubisCO form I (pCC1FOS::cbbLS) and the third encoded the large subunit of form II (pCC1FOS::cbbM). All specific RubisCO activities are summarized in Figure 2a and Supplementary Table S2. Details of genes present on inserts in pCC1FOS clones are shown in Figure 2b.

The specific RubisCO activity of TH-55 was $252 \pm 19 \mathrm{nmol} 3$-PGA $\mathrm{min}^{-1} \mathrm{mg}^{-1}$ in the exponential growth phase (Supplementary Figure S1), which is in the same magnitude of what has been measured for other Thiomicrospira isolates (Takai et al., 2005; Scott et al., 2006). In contrast, the specific RubisCO activity of the TH-55 genomic fosmid clone $6 \mathrm{~F} 3$ was 1.8-fold higher ( $455 \pm 30 \mathrm{nmol} 3$-PGA $\mathrm{min}^{-1} \mathrm{mg}^{-1}$ ). However, this can be explained by the copy number of the pCC1FOS vector: after autoinduction, $\sim 10$ 200 plasmid copies are present in the cell and thus more RubisCO is expressed than in the natural host TH-55. Under the provided conditions, recombinant cbbLS from TH-55 expressed individually in E. coli exhibited a specific RubisCO activity (266 $\pm 23 \mathrm{nmol}$ 3 -PGA min ${ }^{-1} \mathrm{mg}^{-1}$ ), which was $59 \%$ of what we measured for the fosmid clone 6F3 containing the genomic insert from TH-55. The individual expression of the recombinant $c b b M$ from TH-55 in E. coli (55 $\pm 9 \mathrm{nmol} 3$-PGA $\mathrm{min}^{-1} \mathrm{mg}^{-1}$ ) only reached $12 \%$ of the total specific RubisCO activity of fosmid clone 6F3. Since RubisCO form I has a higher specificity for $\mathrm{CO}_{2}$ relative to oxygen and thus a higher tolerance toward oxygen than RubisCO form II (Tabita, 1999), it is not surprising that in our experiments, performed under fully oxic conditions, RubisCO activity of the recombinant RubisCO form I was considerably higher than the activity of RubisCO form II. However, when we added up the individual RubisCO activities measured from pCC1FOS::cbbLS and pCC1FOS::cbbM, still only $71 \%$ of the total RubisCO activity of genomic fosmid clone 6F3 could be reached. This suggests that genes other than the structural genes exist on the genomic fragment that contribute to expressing a fully functional RubisCO. Nevertheless, these experiments showed that the function-based screening can be successfully applied to genomic fosmid clones for identifying recombinant RubisCO active enzymes on a single scale.

Given that single sample screening of thousands of fosmid clones for a specific function is somewhat cumbersome and does not allow searching through a high number of metagenomic clones, we tested whether one active fosmid clone, namely clone 6F3 (455 $\pm 30 \mathrm{nmol} 3$-PGA min $\left.^{-1} \mathrm{mg}^{-1}\right)$, can still be identified if tested together in a pool with other 11 , 23, 47 or 95 inactive clones. A clear detection of RubisCO activity from one active clone among other clones is only guaranteed when no more than 24 clones are pooled for RubisCO activity measurements (Supplementary Figure S2).

\section{Recovering RubisCO active fosmid clones from metagenomic hydrothermal fluid samples}

We then applied our newly established screen for seeking RubisCO active fosmid clones to a metagenomic library that we constructed with DNA from deep-sea hydrothermal vent fluids. From 1056 screened fosmid clones, 12 fosmids exhibited RubisCO activity $\left(427 \pm 16\right.$ to $484 \pm 44 \mathrm{nmol} 3$-PGA $\min ^{-1} \mathrm{mg}^{-1}$ ) (Figure 2a; Supplementary Table S2). Restriction analyses of the corresponding fosmids showed that these 12 clones had $\sim 35.2 \mathrm{~kb}$ DNA inserts and shared the same restriction pattern (data not shown). Doublestranded sequencing of three metagenomic fragments including the RubisCO gene cluster for all 12 clones demonstrated a $100 \%$ DNA identity (for genes encoded on the fragments see Figure $2 b$ and Supplementary Table S3) and resembled genes from T. crunogena XCL-2 (90-96\%) (Scott et al., 2006). Sequencing of one representative clone illustrated that the genes encoded on the DNA metagenomic insert of the fosmid clone followed the arrangement of the homologous genes from XCL-2 (Tcr0411-Tcr0446) (Scott et al., 2006), but with four genes missing (Tcr0415-Tcr0418), namely those encoding an ApbElike lipoprotein (Tcr0416), a hypothetical protein with a FMN-binding domain (Tcr0417) and two hypothetical proteins with unknown function (Tcr0415 and Tcr0418) (Supplementary Figure S3).

Figure 1 Overview of the experimental setup. The experimental setup includes an overview of the experiments conducted to establish the function-based screen, its upscaling and its application to metagenomic libraries. It further illustrates the steps to elucidate the functions of genes recognized on metagenomic fragments recovered by the function-based screen. (a) First, a schematic view of major points successively processed to establish a functional screen to seek RubisCO active clones from crude extracts, and genomic libraries on a single scale is given. (b) The process of upscaling is shown, where pools of 96, 48, 24 and 12 clones were initially applied to test whether one RubisCO active fosmid with TH-55 genomic DNA (clone 6F3) could still be detected among 11, 23, 47 and 95 inactive clones (6F8 from the genomic TH-55 library). Enlargements of the HPLC chromatograms are shown in Supplementary Figure S2. (c) The methodological procedure utilized to screen metagenomic libraries for RubisCO active fosmid clones is shown. (d) Finally, the experiments performed with the recognized RubisCO active metagenomic fragment to elucidate the roles of distinct genes and respective gene products are summarized. A detailed magnification of the $35.2 \mathrm{~kb}$ and $13 \mathrm{~kb}$ gene cluster is shown in Figure 2 . 
TH-55

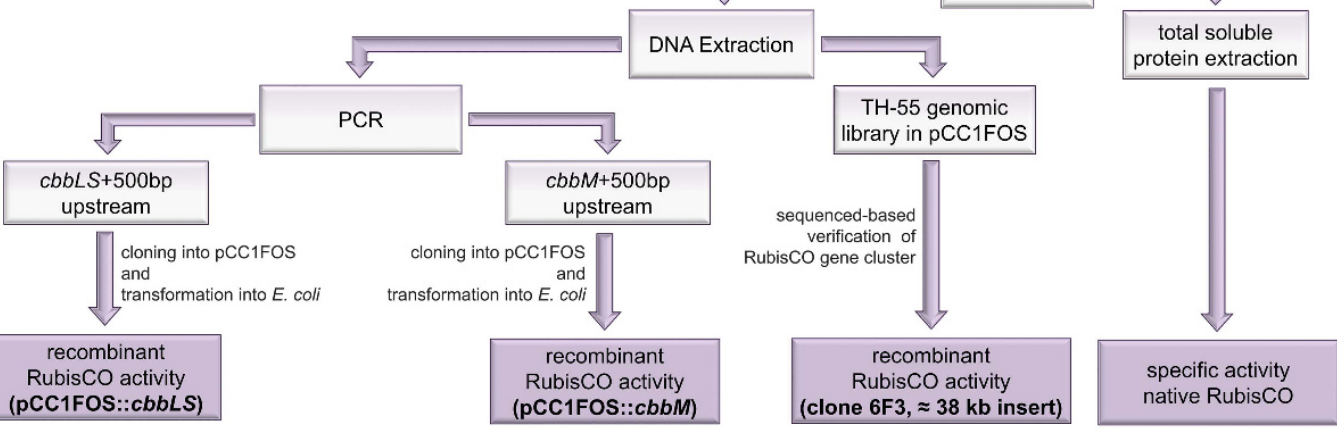

b upscaling the screen - metagenomic scale

microtiterplate loaded with the RubisCO active clone $6 \mathrm{~F} 3$ in one well and the Rubis CO inactive clone 6F8 in all other wells over stamped fosmid clones autoinduction total of pooled $\square$ soluble $\square \quad$ HPLC analyses size 96 pool
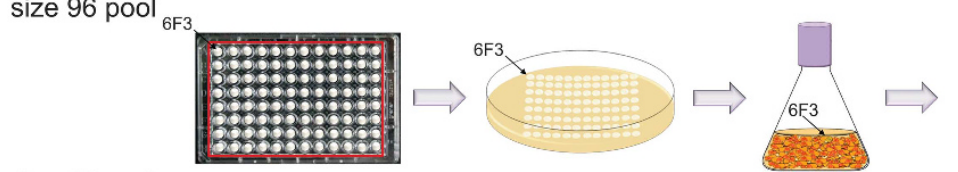

size 48 pool
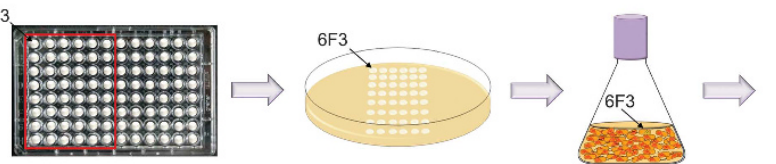

size 24 pool
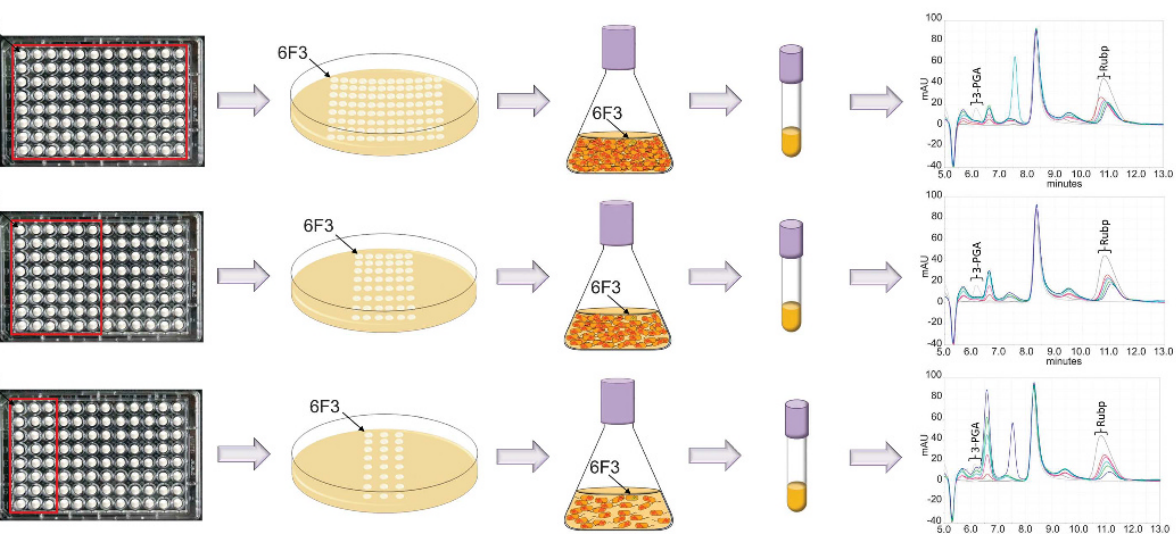

size 12 pool 6 F
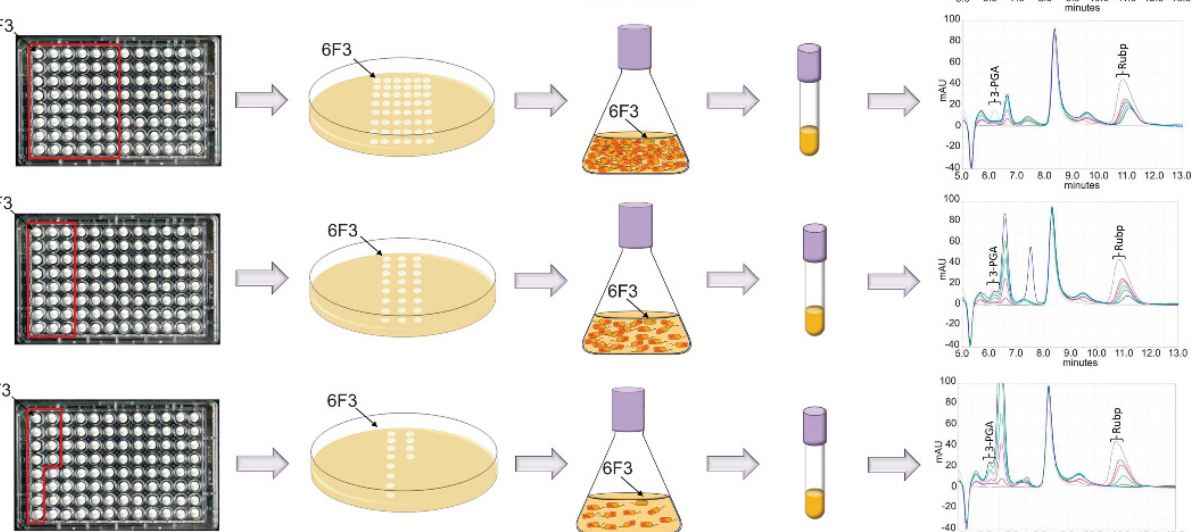

C using the metagenomic screen size 24 pool
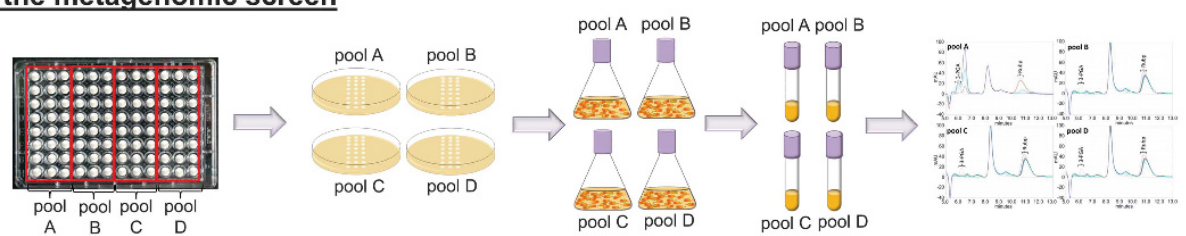

positive tested pools were disrupted and broken down into pools of $2 \times 12,3 \times 4$ and $4 \times 1$ respectively, to identify the RubisCO active clone

\section{d investigating metagenome derived RubisCOs}

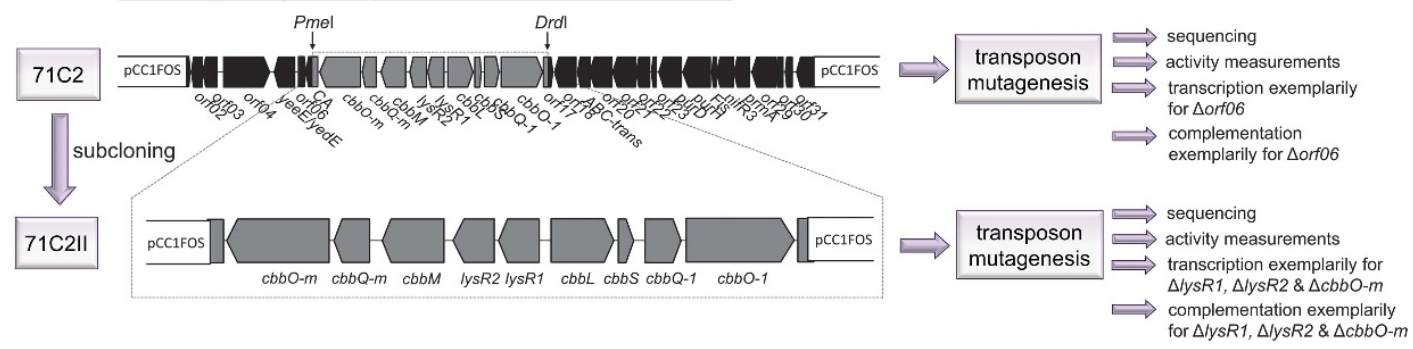




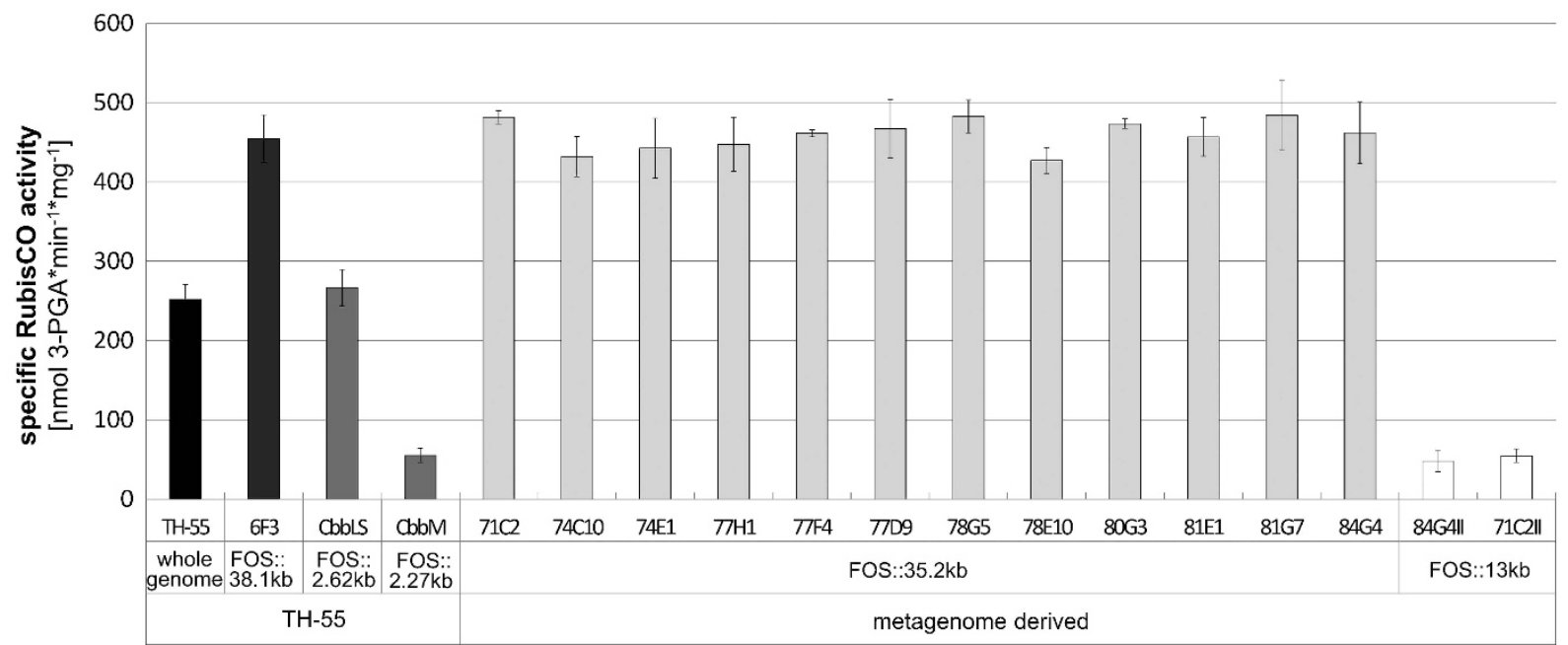

b

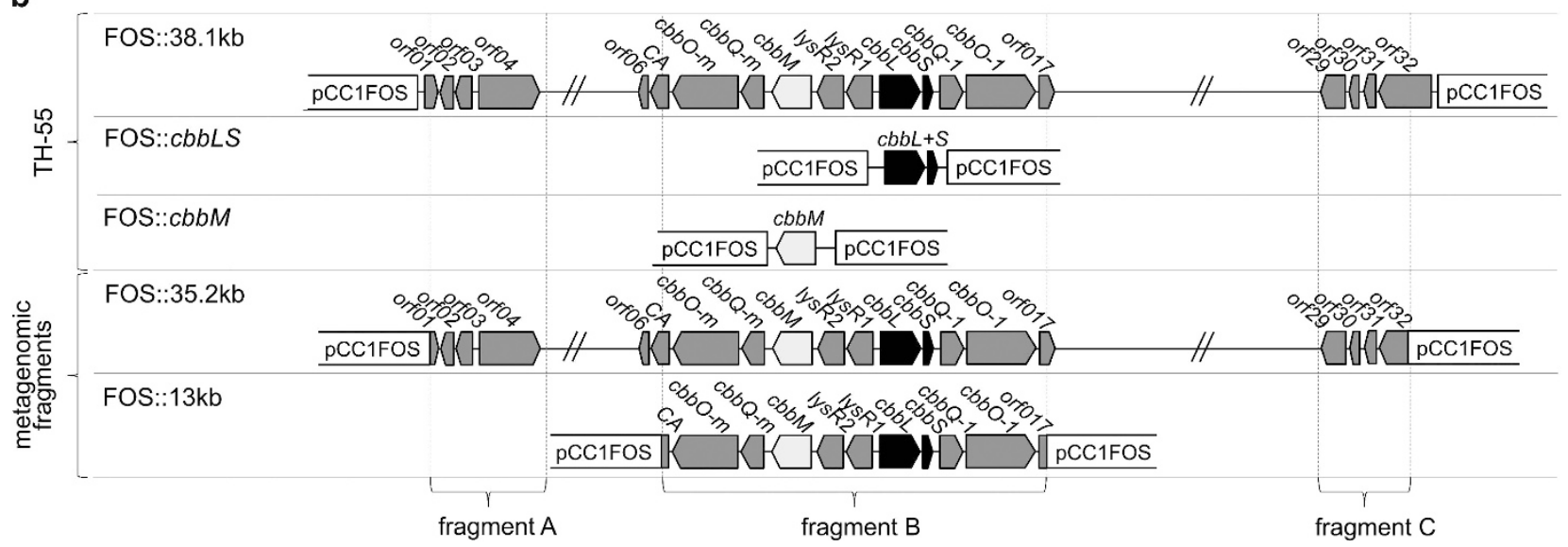

Figure 2 Specific RubisCO activities of TH-55 and recombinant RubisCOs as well as respective gene arrangements. (a) Specific RubisCO activities measured from crude extracts of TH-55 (TH-55, grown for $25 \mathrm{~h}$ ), clone $6 \mathrm{~F} 3$ ( $38.1 \mathrm{~kb}$ insert size) derived from a genomic fosmid library constructed with TH-55 DNA (FOS::38.1 kb), a pCC1FOS clone containing TH-55's cbbLS structural genes (FOS::cbbLS), a pCC1FOS clone containing TH-55's $c b b M$ structural gene (FOS::cbbM), fosmid clones derived from the Nibelungen metagenome library (71C2, 74C10, 74E1, 77H1, 77F4, 77D9, 78G5, 78E10, 80G3, 81E1, 81G7 and 84G4, summarized as FOS::35.2 kb) and fosmid subclones (84G4II and 71C2II) containing the RubisCO gene cluster (fragment B; $13 \mathrm{~kb}$ ) (summarized as FOS::13 kb). (b) Schematic representation of the gene arrangements of the DNA inserts. Identified open reading frames are indicated as gray arrows and structural RubisCO genes are indicated in black or light gray, for $c b b L S$ or $c b b M$, respectively, in the direction of transcription. ORFs were numbered serially from orfo1 to orf32 (for details on annotations of respective ORFs see Supplementary Table S3). Standard gene abbreviations were used (see Supplementary Table S6). Fragments A, B and C denote regions that were sequenced for all 12 RubisCO active metagenome-derived fosmid clones sought from the Nibelungen library through the function-based RubisCO screen.

Transposon insertion libraries to elucidate the role of metagenomic gene products on RubisCO activity

To investigate whether additional enzymes other than the structural enzymes CbbLS and CbbM may be required for a fully functional RubisCO, we subcloned the RubisCO gene cluster (13 kb) of two metagenomic clones (71C2 and clone 84G4) and measured their specific RubisCO activity. The specific RubisCO activities of these two subclones 71C2II and 84G4II were roughly fivefold lower ( $55 \pm 8$ and $48 \pm 14 \mathrm{nmol} 3$-PGA min ${ }^{-1} \mathrm{mg}^{-1}$ ) than the activity measured for the respective clones $71 \mathrm{C} 2$ and $84 \mathrm{G} 4$ with the original metagenomic DNA fragment containing flanking regions of additional
$22.2 \mathrm{~kb}$ DNA (Figure 2; Supplementary Table S2). This is suggestive of genes up- and/or downstream of the cloned RubisCO gene cluster contributing to the expression of a fully functional RubisCO enzyme.

To unravel which genes and respective products in and outside of the RubisCO gene cluster contribute to a fully functional RubisCO in our metagenomic clones, two transposon mutant libraries were constructed: one with the fosmid clone $71 \mathrm{C} 2$ (with the entire metagenomic DNA fragment including the RubisCO gene cluster and flanking DNA regions: $35.2 \mathrm{~kb}$ ) and one with the fosmid clone 71C2II (carrying only the RubisCO 
gene cluster: $13 \mathrm{~kb}$ ). In total, we sequenced 384 transposon clones with a primer targeting the transposase-inserted kanamycin cassette to establish the position of the insertion and measured RubisCO activity for 45 selected transposon clones (Supplementary Tables S4 and S5). Additionally, we performed complementation experiments with four transposon clones exhibiting the most dramatic activity changes (not related to structural RubisCOs), to show that the original RubisCO activity could be restored. In the complementation experiments, some discrepancies to the full activity of the intact fragments are visible. However, they are most likely related to the diverging and irregular copy number of pCC1FOS (after autoinduction, $10+$ fosmid copies) and pUC19 (without any induction, $100+$ plasmid copies). We further linked the most dramatic activity change of four transposon clones with transcription of RubisCO forms I and II to elucidate whether these deleted genes are involved in transcription or rather in subsequent cellular processes.

\section{The effect of gene deletions outside of the RubisCO} gene cluster on RubisCO activity

Among the 25 transposon clones with deletions upor downstream of the $13 \mathrm{~kb}$ RubisCO gene cluster, seven clones had significantly lower RubisCO activity than the intact $71 \mathrm{C} 2$ clone (Figures 3a and b). One transposon clone, namely clone 96, exhibited a dramatic activity loss. The activity was comparable to the reduced RubisCO activity measured for the $13 \mathrm{~kb}$ clones, which had the RubisCO gene cluster but lacked the flanking DNA regions (Figure 2a; Supplementary Table S2). The gene deleted in transposon clone 96 (insertion at 6 of $135 \mathrm{aa})$ was designated orf06 and resembled the gene Tcr0420 of XCL-2 $(97 \%$ aa similarity $)$ (Supplementary Table S3). In XCL-2 this gene product has been assigned a hypothetical protein with unknown function (Scott et al., 2006). Interestingly, the closest related protein of known function to orf06's gene product was the ArsR regulatory protein of Micromonospora aurantiaca ATCC 27029 (Bioproject: PRJNA42501) (Supplementary Figure S4) (30\% aa identity), which functions as a metal-sensing transcriptional repressor (Wu and Rosen, 1991). Transcription experiments with the transposon clone 96 ( $\Delta$ orfo6) where the relative abundance of $c b b L$ and $c b b M$ gene products was determined, demonstrated that transcription for both genes is downregulated relative to the metagenomic fragment with the intact orfo6 (Supplementary Figure S5). Conclusively, the orf06 gene product is likely involved in directly or indirectly regulating the structural RubisCO forms I and II. Complementation experiments of transpo-

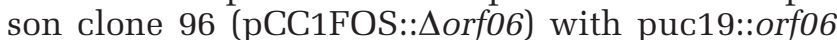
managed to restore most of the original RubisCO activity (Supplementary Figure S6), evidencing that orf06 is responsible for the dramatic activity loss. We also conducted experiments with TH-55's RNA to identify which genes were co-transcribed. They showed that in TH-55 the orf06 homolog was transcribed separately from the carbonic anhydrase and other neighboring RubisCO-associated genes (Figure 4; Supplementary Figure S7). The activity loss of transposon clone 231, with an insertion in the non-coding region upstream of orfo6 (Figures 3a and b), may be related to difficulties in the transcription process of orfo6 due to an impaired promoter region.

Other transposon clones with insertions outside of the RubisCO gene cluster included clones 58, 89, 68 and 91 (for details see Supplementary Results). Despite RubisCO activity loss, none of these four enzymes have ever been linked to the expression of a fully functional RubisCO and current annotations do not imply that these genes encode functions associated with RubisCO regulation, assembling or activation mechanisms. However, the significant RubisCO activity loss may be explained if gene products interplay with RubisCO-associated genes encoded on the entire metagenomic fragment or if the activity of encoded enzymes has an impact on intercellular conditions such as $\mathrm{CO}_{2}$ concentrations, $\mathrm{pH}$ values or ATP/ADP ratio, which are important to maximize RubisCO activity (Portis, 1990; Toyoda et al., 2005) and which might be applicable in heterologous hosts like E. coli as well.

The effect of gene deletions within the RubisCO gene cluster on RubisCO activity

Among the 20 transposon clones exhibiting deletions in the RubisCO gene cluster, 10 displayed a significantly lower and two clones a significantly higher RubisCO activity relative to the respective intact fosmid clone (Figure 3). An insertion in the transposon clone 149II resulted in a significant increase in RubisCO activity (Figures 3c and d). Sequencing of this gene (orf11) revealed a similarity to lysR2 of the LysR family, which is classified as a transcriptional regulator (Maddocks and Oyston, 2008). The insertion in orf11 was located at base 553 (of $945 \mathrm{nt}$ ), that is, 185 aa (of 315 aa). Blastp of the translated intact orf11 of the metagenomic fosmid clone demonstrated that the LysR substrate binding domain is located between 99 and 303 aa, and thus a considerable impact on the LysR binding ability can be expected through the deletion, which can explain the significant change in RubisCO activity. According to the transcription experiments with transposon clone $149 \mathrm{II}$ ( $\Delta$ lysR2), it appears that the transcription level of $c b b L$ remained unchanged but that transcription was upregulated for $c b b M$ relative to the respective intact metagenomic fragment (Supplementary Figure S5), suggesting that Orf11 acts on $c b b M$ transcription as a repressor. In complementation experiments with the transposon clone 149II (pCC1FOS:: $\operatorname{lys} R 2$ ) and pUC19::lysR2 


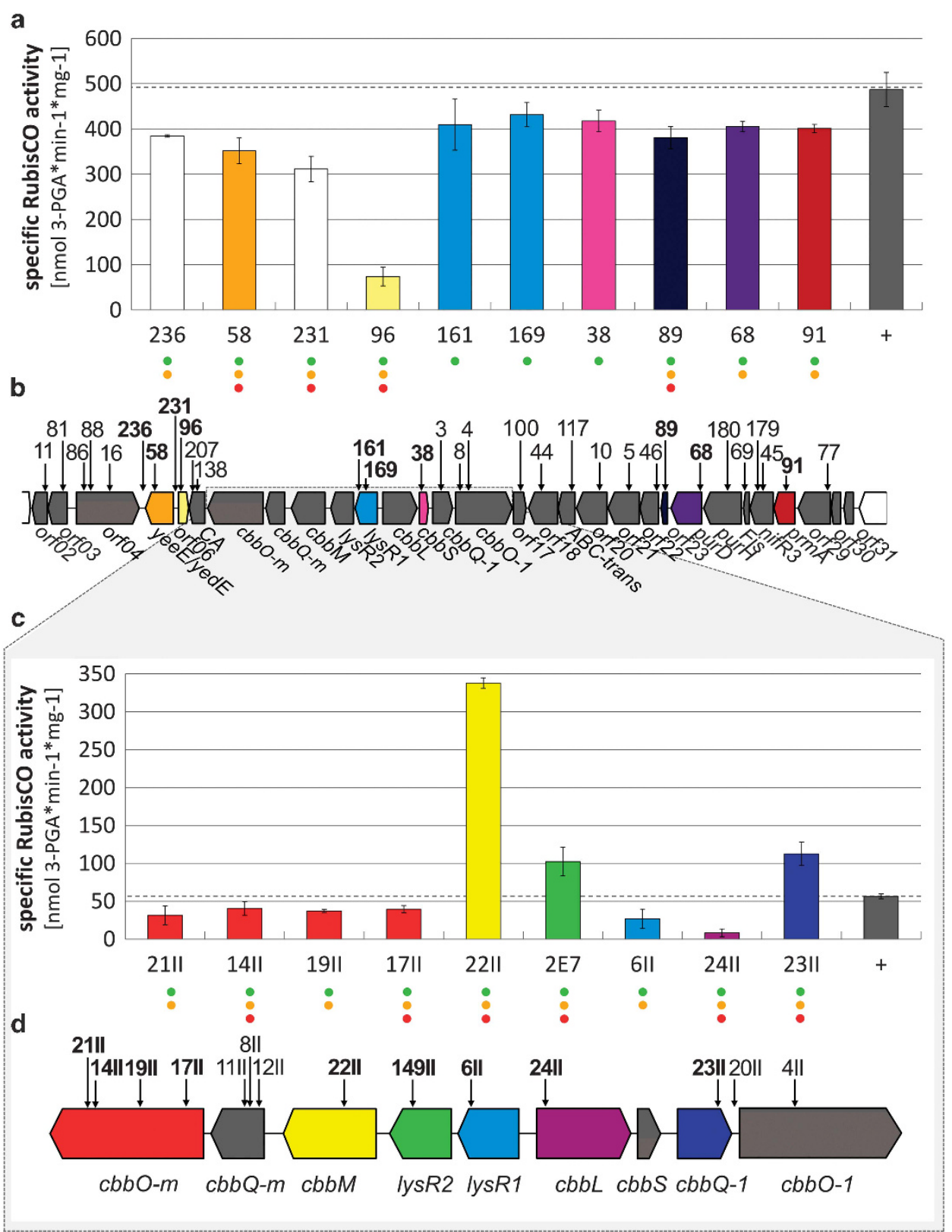

Figure 3 Specific RubisCO activities and insertion positions of tested transposon clones. Specific RubisCO activities (a) and schematic gene arrangement (b) of transposon clones carrying the entire $35.2 \mathrm{~kb}$ insert derived from the metagenomic fosmid clone $71 \mathrm{C} 2$. Specific RubisCO activities (c) and schematic gene arrangement (d) of transposon clones carrying the $13 \mathrm{~kb}$ insert derived from the fosmid clone 71C2II. Only specific RubisCO activities for transposon clones with significant differences to the intact $35.2 \mathrm{~kb}$ (a) or $13 \mathrm{~kb}$ (c) fosmid clones (intact fosmid clones indicated as +) are displayed. Identified ORFs were numbered consecutively, designated orf01-orf32 (for detailed abbreviations see Supplementary Table S3) and indicated as arrows in the direction of transcription. Insertion sites are denoted by vertical black arrows and transposon clone numbers. Clones exhibiting significant changes in RubisCO activity relative to the intact clones are highlighted in bold. Genes with significant activity change are color coded according to the bars in a or c. The level of significance is denoted by dots under the activity, where green is $0.05(95 \%)$, yellow is $0.01(99 \%)$ and red is $0.001(99.9 \%)$.

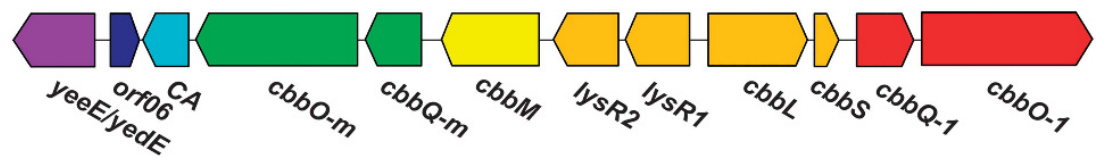

Figure 4 Transcription of RubisCO encoding genes and flanking gene regions from TH-55. Genes are indicated as arrows. Co-transcribed genes are displayed in the same color. Abbreviations are listed in Supplementary Table S7. 
the RubisCO activity dropped down near the level of the intact metagenomic fragment (Supplementary Figure S6).

In contrast, three insertions in orf12 (lysR1) at the nucleotide positions (i) 660, (ii) 790 and (iii) 855 (of $927 \mathrm{nt}$ ), conferring to aa positions (i) 220, (ii) 264 and (iii) 285 (of total 308 aa), led to significant lower activities for clones (i) 169, (ii) 6II and (iii) 161, respectively, relative to the intact metagenomic clones (Figures 3a and c). Complementation experi-

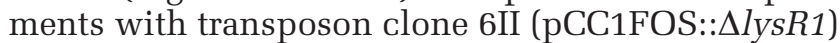
and pUC19::lysR1 managed to restore the original RubisCO activity of the intact metagenomic fragment (Supplementary Figure S6). Blastp searches of the translated, intact metagenomic orf12 gene suggest that the LysR substrate binding domain is located between 93 and 298 aa and thus the insertions affect the last (i) 78 aa, (ii) 34 and 13 aa of the LysR substrate binding domain of the metagenomic transposon clones. Transcription experiments with transposon clone 6II ( $\Delta$ lysR1) suggest an unchanged transcription level for $c b b L$ but a downregulation of $c b b M$ relative to the respective intact metagenomic fragment (Supplementary Figure S5). We thus propose that orf12 (LysR1 homolog) activates $c b b M$ transcription. However, Toyoda et al. (2005) suggested that in $H$. marinus transcriptional regulators of the lysR family, namely CbbR1 and CbbRm, were required to activate the expression of $c b b L S-1$ and $c b b M$, respectively, which in our case only holds true for orf12 (LysR1 homolog) and cbbM. More experiments will be needed to better understand the interaction and interconnection between LysR regulators and the structural RubisCOs. Transcription experiments with TH-55 suggest that lysR2, lysR1 and cbbLS were co-transcribed (Figure 4; Supplementary Figure S7). Yet, since the lysR2 and lysR 1 genes are juxtaposed to $c b b L S$, either only the lysR 2 and lysR 1 or the $c b b L S$ gene product would result in a functioning enzyme. Nevertheless, if the promoter regions of the lysRs and the structural gene $c b b L$ overlap, which is typical for lysR genes, which regulate their own transcription and that of adjacent structural genes (Schell, 1993; Maddocks and Oyston, 2008), the one acquired transcript (lysR2R1cbbLS) might mask two actual transcripts (that is, lysR2R1 and $c b b L S$ ).

As the role that CbbO and CbbQ play for a fully functional RubisCO remains unclear, we selected 12 transposon clones with insertions scattered across orfo8 (cbbO-m), orfo9 (cbbQ-m), orf16 (cbbO-1) and orf15 (cbbQ-1) of our metagenomic fragment for RubisCO activity measurements. Four transposon clones with insertions at different positions in cbbO-m had a significant activity loss (Figures 3c and d). The full RubisCO activity could be restored when transposon clone 17II (pCC1FOS:: $\Delta c b b O-m)$ was complemented with pUC19::cbbO-m (Supplementary Figure S6). In the transposon clone 17II $(\Delta c b b O-m)$, transcription of $c b b L$ and $c b b M$ was maintained at an unchanged level relative to the intact metagenomic clone (Supplementary Figure S5). Therefore, it appears highly likely that CbbO-m is not involved in regulating transcription of the structural RubisCO genes but rather in post-transcriptional processes, as has been suggested (Hayashi et al., 1997, 1999; Scott et al., 2006). No changes in RubisCO activity were evident in $\triangle c b b Q-$ $m$ (8II, $11 \mathrm{II}$ and 12II) and $\Delta c b b O-1$ (4, 8 and 4II) transposon clones compared to the intact metagenomic clone 71C2II (pCC1FOS::13 kb) or $71 \mathrm{C} 2$ (pCC1FOS::35.2 kb). Mutations in CbbQ-m and CbbO-1 may be able to be substituted by CbbQ-1 and CbbO-m functioning, respectively, or these enzymes may simply not be as detrimental for producing a fully functional RubisCO. Curiously, two transposon clones with deletions in orf15 $(\Delta c b b Q-1)$ exhibited contrasting results for the RubisCO activity. Transposon clone 23II carrying the RubisCO gene cluster $(13 \mathrm{~kb})$ had significantly higher activities relative to the intact metagenomic clone, but the RubisCO activity of transposon clone 3 with the entire metagenomic DNA fragment $(35.2 \mathrm{~kb})$ remained stable (Figure 3$)$. Although these results appear contrasting at first glance they can be explained: $c b b Q-1$ may need to interact with proteins encoded on the flanking DNA regions. Interestingly, in TH-55 neither $c b b Q O-m$ nor $c b b Q O-$ 1 was co-transcribed with $c b b M$ or $c b b L S$ (Figure 4; Supplementary Figure S7).

In transposon clones with insertions in the structural genes $c b b S$ (clone 38) and $c b b L$ (clone 24II), RubisCO activity diminished significantly relative to the intact metagenomic clone 71C2II (pCC1FOS::13 kb) or 71C2 (pCC1FOS::35.2 kb) (Figure 3). On the contrary, an insertion in the RubisCO form II structural gene $c b b M$ (clone 22II) led to an increased RubisCO activity (6.2-fold higher) relative to the intact version of clone 71C2II (pCC1FOS::13 kb), indicating that RubisCO form II ( $c b b M)$ may repress RubisCO form I ( $c b b L)$. According to transcription experiments with RNA from TH-55, $c b b L S$ was transcribed separately from cbbM (Figure 4; Supplementary Figure S7).

\section{Conclusion}

Although novel enzymes can only be discovered by function-based screening approaches, the imperative of heterologously expressing environmental DNA from an unknown source organism in a culturable surrogate host is an inherent limitation itself and often associated with low hit rates (Perner et al., 2011 and references therein). It has been illustrated that only $30-40 \%$ of foreign DNA are successfully expressed in E. coli (Rondon et al., 1999; Gabor et al., 2004), the host we used for our screen. Some of the problems faced in recombinant protein expression in E. coli include recognition of intrinsic promoters and associated factors, diverging codon usage, translation, correct folding, 
maturation processes and activation of the enzyme (cf. Perner et al., 2011 and references therein). Since we work with large metagenomic DNA fragments (average insert size of our fosmid clones $37 \pm 2.1 \mathrm{~kb}$ ), it can be expected that promoters and ribosomal binding sites of the targeted genes are located on the metagenomic fragment. All the RubisCO active clones we discovered resembled genes of Thiomicrospira, which could indicate the high abundance of Thiomicrospira in the analyzed sample. However, these findings may also indicate the difficulties of our screen in expressing RubisCOs from other organisms. For example, RubisCOs of taxonomically distinct species or anaerobic organisms may not be detectable in the host $E$. coli under the provided experimental conditions. Further experiments will be needed to recognize the full extent of RubisCOs that can be targeted. Despite possible limitations that may exist when applying this screen, we show that it can be successfully used to identify RubisCO active fosmid clones from a metagenomic library and that these clones can be utilized to elucidate the importance of flanking genes and the respective enzymes for a fully functional RubisCO. Our approach circumvents time-consuming cultivation of autotrophic microorganisms, some of which may be resilient to current cultivation techniques, and the inherent bias accompanying sequence-dependent methods. It allows us to tap the tremendous metagenomic resource available in the environment and hence enables the discovery of species not previously associated with RubisCO activity.

\section{Conflict of Interest}

The authors declare no conflict of interest.

\section{Acknowledgements}

We thank the captain and crews of the RV Meteor as well as the ROV Kiel6000 (GEOMAR, Kiel) for helping us to obtain deep-sea vent samples. We thank Wenke Bahnsen and Nicole Adam for excellent technical assistance in the laboratory and Wolfgang $\mathrm{R}$ Streit for stimulating discussions and suggestions during the work. This work was supported by grants from the Deutsche Forschungsgemeinschaft (DFG) (PE1549/5-1) and the DFG priority program 1144 'From Mantle to Ocean: Energy-, Materialand Life-cycles at Spreading Axes'.

\section{References}

Berg IA. (2011). Ecological aspects of the distribution of different autotrophic $\mathrm{CO}_{2}$ fixation pathways. Appl Environ Microbiol 77: 1925-1936.

Bradford MM, Williams WL. (1976). New, rapid, sensitive method for protein determination. Fed Proc 35: 274-274.
Dobrinski KP, Longo DL, Scott KM. (2005). The carbon concentrating mechanism of the hydrothermal vent chemolithoautotroph Thiomicrospira crunogena. J Bacteriol 187: 5761-5766.

Ellis RJ. (1979). Most abundant protein in the world. Trends Biochem Sci 4: 241-244.

Field CB, Behrenfeld MJ, Randerson JT, Falkowski P. (1998). Primary production of the biosphere: integrating terrestrial and oceanic components. Science 281: 237-240.

Gabor EM, Alkema WB, Janssen DB. (2004). Quantifying the accessibility of the metagenome by random expression cloning techniques. Environ Microbiol 6: 879-886.

Hayashi NR, Arai H, Kodama T, Igarashi Y. (1997). The novel genes, $c b b Q$ and $c b b O$, located downstream from the RubisCO genes of Pseudomonas hydrogenothermophila, affect the conformational states and activity of RubisCO. Biochem Biophys Res Commun 241: 565-569.

Hayashi NR, Arai H, Kodama T, Igarashi Y. (1999). The $c b b Q$ genes, located downstream of the form I and form II RubisCO genes, affect the activity of both RubisCOs. Biochem Biophys Res Commun 265: 177-183.

Jakob R, Saenger W. (1985). Reversed phase ion pair chromatographic separation of ribulose-1,5-bisphosphate from 3-phosphoglycerate and its application as a new enzyme assay for Rubp carboxylase oxygenase. FEBS Lett 183: 111-114.

Jannasch HW, Wirsen CO, Nelson DC, Robertson LA. (1985). Thiomicrospira crunogena sp. nov. a colorless, sulfur-oxidizing bacterium from a deep-sea hydrothermal vent. Int J Syst Bacteriol 35: 422-424.

Joshi GS, Romagnoli S, Verberkmoes NC, Hettich RL, Pelletier D, Tabita FR. (2009). Differential accumulation of form I RubisCO in Rhodopseudomonas palustris CGA010 under photoheterotrophic growth conditions with reduced carbon sources. J Bacteriol 191: 4243-4250.

Kusian B, Bowien B. (1997). Organization and regulation of $c b b \mathrm{CO}_{2}$ assimilation genes in autotrophic bacteria. FEMS Microbiol Rev 21: 135-155.

Li LA, Gibson JL, Tabita FR. (1993). The Rubisco activase (rca) gene is located downstream from $r b c S$ in Anabaena sp. strain CA and is detected in other Anabaena/Nostoc strains. Plant Mol Biol 21: 753-764.

Maddocks SE, Oyston PCF. (2008). Structure and function of the LysR-type transcriptional regulator (LTTR) family proteins. Microbiology 154: 3609-3623.

Melchert B, Devey CW, German CR, Lackschewitz KS, Seifert R, Walter M et al. (2008). First evidence for high-temperature off-axis venting of deep crustal/ mantle heat: the Nibelungen hydrothermal field, southern Mid-Atlantic Ridge. Earth Planet Sci Lett 275: 61-69.

Mueller-Cajar O, Stotz M, Wendler P, Hartl FU, Bracher A, Hayer-Hartl M. (2011). Structure and function of the $\mathrm{AAA}+$ protein CbbX, a red-type Rubisco activase. Nature 479: 194-199.

Perner M, Seifert R, Weber S, Koschinsky A, Schmidt K, Strauss $\mathrm{H}$ et al. (2007). Microbial $\mathrm{CO}_{2}$ fixation and sulfur cycling associated with low-temperature emissions at the Lilliput hydrothermal field, southern Mid-Atlantic Ridge (9.S). Environ Microbiol 9: 1186-1201. 
Perner M, Bach W, Hentscher M, Koschinsky A, Garbe-Schonberg D, Streit WR et al. (2009). Short-term microbial and physico-chemical variability in low-temperature hydrothermal fluids near $5^{\circ} \mathrm{S}$ on the Mid-Atlantic Ridge. Environ Microbiol 11: 2526-2541.

Perner M, Ilmberger N, Köhler HU, Chow J, Streit WR. (2011). Emerging fields in functional metagenomics and its industrial relevance - overcoming limitations and redirecting the search for novel biocatalysts. In de Bruijn FJ (ed) Handbook of Molecular Microbial Ecology II: Metagenomics in Different Habitats. John Wiley \& Sons, Inc.: Hoboken, NJ.

Perner M, Hansen M, Seifert R, Strauss H, Koschinsky A, Petersen S. (2013). Linking geology, fluid chemistry, and microbial activity of basalt- and ultramafic-hosted deep-sea hydrothermal vent environments. Geobiology 11: $340-355$.

Portis AR Jr. (1990). Rubisco activase. Biochim Biophys Acta 1015: 15-28.

Portis AR Jr. (2003). Rubisco activase - Rubisco’s catalytic chaperone. Photosynth Res 75: 11-27.

Raven JA. (2009). Contributions of anoxygenic and oxygenic phototrophy and chemolithotrophy to carbon and oxygen fluxes in aquatic environments. Aquat Microb Ecol 56: 177-192.

Raven JA. (2013). Rubisco: still the most abundant protein of Earth? New Phytol 198: 1-3.

Rondon M, Raffel S, Goodman R, Handelsman J. (1999). Toward functional genomics in bacteria: analysis of gene expression in Escherichia coli from a bacterial artificial chromosome library of Bacillus cereus. Proc Natl Acad Sci USA 96: 6451-6455.

Schell MA. (1993). Molecular biology of the LysR family of transcriptional regulators. Annu Rev Microbiol 47: 597-626.

Schwedock J, Harmer TL, Scott KM, Hektor HJ, Seitz AP, Fontana MC et al. (2004). Characterization and expression of genes from the RubisCO gene cluster of the chemoautotrophic symbiont of Solemya velum: cbbLSQO. Arch Microbiol 182: 18-29.

Scott KM, Sievert SM, Abril FN, Ball LA, Barrett CJ, Blake RA et al. (2006). The genome of deep-sea vent chemolithoautotroph Thiomicrospira crunogena XCL-2. PLoS Biol 4: 2196-2212.

Tabita FR. (1999). Microbial ribulose-1,5-bisphosphate carboxylase/oxygenase: a different perspective. Photosynth Res 60: 1-28.

Tabita FR, Hanson TE, Li H, Satagopan S, Singh J, Chan S. (2007). Function, structure, and evolution of the RubisCO-like proteins and their RubisCO homologs. Microbiol Mol Biol Rev 71: 576-599.

Tabita FR, Satagopan S, Hanson TE, Kreel NE, Scott SS. (2008). Distinct form I, II, III, and IV Rubisco proteins from the three kingdoms of life provide clues about Rubisco evolution and structure/function relationships. J Exp Bot 59: 1515-1524.

Takai K, Campbell BJ, Cary SC, Suzuki M, Oida H, Nunoura $\mathrm{T}$ et al. (2005). Enzymatic and genetic characterization of carbon and energy metabolisms by deep-sea hydrothermal chemolithoautotrophic isolates of Epsilonproteobacteria. Appl Environ Microbiol 71: 7310-7320.

Toyoda K, Yoshizawa Y, Arai H, Ishii M, Igarashi Y. (2005). The role of two CbbRs in the transcriptional regulation of three ribulose-1,5-bisphosphate carboxylase/oxygenase genes in Hydrogenovibrio marinus strain $\mathrm{MH}-$ 110. Microbiology 151: 3615-3625.

Witte B, John D, Wawrik B, Paul JH, Dayan D, Tabita FR. (2010). Functional prokaryotic RubisCO from an oceanic metagenomic library. Appl Environ Microbiol 76: 2997-3003.

Wu J, Rosen BP. (1991). The ArsR protein is a trans-acting regulatory protein. Mol Microbiol 5: 1331-1336.

Xie W, Wang F, Guo L, Chen Z, Sievert SM, Meng J et al. (2011). Comparative metagenomics of microbial communities inhabiting deep-sea hydrothermal vent chimneys with contrasting chemistries. ISME J 5: 414-426.

Supplementary Information accompanies this paper on The ISME Journal website (http://www.nature.com/ismej) 